\title{
Can radiative decay of long-lived particles after the BBN solve cosmological ${ }^{6}$ Li problem?
}

\author{
Motohiko Kusakabe ${ }^{*}$ and Toshitaka Kajino \\ Department of Astronomy, School of Science, University of Tokyo and National Astronomical \\ Observatory of Japan

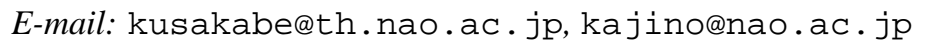

\section{Grant J. Mathews}

Center for Astrophysics, University of Notre Dame

E-mail: 'gmathews@nd. edui'

Recent spectroscopic observations of metal poor stars have indicated that both ${ }^{7} \mathrm{Li}$ and ${ }^{6} \mathrm{Li}$ have abundance plateaus with respect to the metallicity. Abundances of ${ }^{7} \mathrm{Li}$ are about a factor three lower than the primordial abundance predicted by standard big-bang nucleosynthesis (SBBN), and ${ }^{6} \mathrm{Li}$ abundances are $\sim 1 / 20$ of ${ }^{7} \mathrm{Li}$, whereas SBBN predicts negligible amounts of ${ }^{6} \mathrm{Li} \mathrm{com}$ pared to the detected level. These discrepancies suggest that ${ }^{6} \mathrm{Li}$ has another cosmological or Galactic origin than the SBBN. Furthermore, it could appear that ${ }^{7} \mathrm{Li}$ (and also ${ }^{6} \mathrm{Li}$ ) has been depleted from its primordial abundance by some post-BBN processes. We study the possibility that the radiative decay of long-lived particles has affected the cosmological lithium abundances. We calculate the non-thermal nucleosynthesis associated with the radiative decay, and explore the allowed region of the parameters specifying the properties of long-lived particles. We also impose constraints from observations of the CMB energy spectrum. It is found that non-thermal nucleosynthesis produces ${ }^{6} \mathrm{Li}$ at the level detected in metal poor halo stars (MPHSs), when the lifetime of the unstable particles is of the order $\sim 10^{8}-10^{12} \mathrm{~s}$ and their initial abundance with respect to that of the photons is $\sim\left(10^{-13}-10^{-12} \mathrm{GeV}\right) / E_{\gamma 0}$, where $E_{\gamma 0}$ is the emitted photon energy in the radiative decay. We conclude that a combination of two different processes could explain the lithium isotopic abundances in MPHSs. First, a non-thermal cosmological nucleosynthesis associated with the radiative decay of unstable particles; and second, about the same degree of stellar depletion of both primordial lithium isotopic abundances. If MPHSs experience ${ }^{6} \mathrm{Li}$ depletion of factor much greater than $\sim 3$, the simple radiative decay process can not be the cause of large ${ }^{6} \mathrm{Li}$ abundances in MPHSs.

PACS: $26.35 .+\mathrm{C}, 95.35 .+\mathrm{d}, 98.80 . \mathrm{Cq}, 98.80 . \mathrm{Es}$

International Symposium on Nuclear Astrophysics - Nuclei in the Cosmos - IX

25-30 June 2006

CERN

\footnotetext{
*Speaker.

${ }^{\dagger}$ Research Fellow of the Japan Society for the Promotion of Science
} 


\section{Introduction}

In standard cosmology, the universe is thought to have experienced big-bang nucleosynthesis (BBN) at a very early stage. $\mathrm{D}, \mathrm{T},{ }^{3} \mathrm{He},{ }^{4} \mathrm{He},{ }^{6} \mathrm{Li},{ }^{7} \mathrm{Li}$ and ${ }^{7} \mathrm{Be}$ are produced in appreciable amount at this epoch. The Wilkinson Microwave Anisotropy Probe (WMAP) satellite has measured the temperature fluctuations of the cosmic microwave background (CMB) radiation, and parameters characterizing the standard big bang cosmology have been deduced [1, in the baryon-to-photon ratio $\eta$ deduced from fits to the $\mathrm{CMB}$, the $\mathrm{BBN}$ model predicts abundances of the light elements which are more-or-less consistent with those inferred from astronomical observations. This agreement places significant limits on non-standard models which influence the cosmic nuclear abundances.

In this regard, unstable massive particles decaying or annihilating during or after the BBN epoch are strongly constrained [1; hadronic showers which lead to the destruction of preexisting nuclei and to the production of different nuclear species. In turn, these modifications to the light element abundances are used to constrain theories for the decay of relic particles.

Spectroscopic lithium abundances have been detected in the atmospheres of metal poor stars. Nearly constant abundances of ${ }^{6} \mathrm{Li}$ and ${ }^{7} \mathrm{Li}$ in metal-poor Population II (Pop II) stars have been

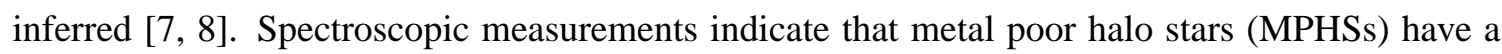
very large abundance of ${ }^{6} \mathrm{Li}$, i.e. at a level of about a twentieth that of ${ }^{7} \mathrm{Li}$. This is about three orders of magnitude larger than the SBBN prediction of the ${ }^{6} \mathrm{Li}$ abundance.

We calculate the nucleosynthesis triggered by the radiative decay processes of long-lived relic particles. We take into account the primary, secondary, and tertiary processes resulting from the electromagnetic cascade showers which both produce and destroy the light elements. We then constrain the abundance of long-lived particles from the calculated nucleosynthesis. We do not find, however, a simultaneous solution to both the ${ }^{7} \mathrm{Li}$ and ${ }^{6} \mathrm{Li}$ abundances unless there is stellar destruction of lithium. We conclude that our model can explain the desired ${ }^{6} \mathrm{Li}$ production by non-thermal nucleosynthesis if there is stellar destruction of factor $\sim 3$ for both lithium isotopes to explain the observed ${ }^{7} \mathrm{Li}\left[\overline{9} \underline{9}^{9}\right]$.

\section{Model}

We assume the creation of high energy photons from the radiative decay of a massive particle

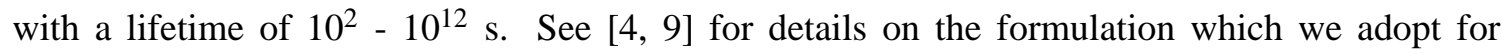
calculation of the non-thermal nucleosynthesis triggered by the high energy non-thermal photons.

We assume that the decaying dark particle is non-relativistic, and almost at rest in the expanding universe. We denote the imaginary particle by $X$, with a mass $M_{X}$ and a life $\tau_{X}$ that decays into a photon plus another dark-matter particle. We represent the emitted photon energy by $E_{\gamma 0}$ and define $\zeta_{X}=\left(n_{X}^{0} / n_{\gamma}^{0}\right) E_{\gamma 0}$, where $\left(n_{X}^{0} / n_{\gamma}^{0}\right)$ is equal to a number ratio of $X$ to photon before $X$-decay. When an energetic photon emerges, it interacts with the cosmic background and induces an electromagnetic cascade shower. The faster processes are pair production through background photons $\gamma_{\mathrm{bg}}\left(\gamma \gamma_{\mathrm{bg}} \rightarrow e^{+} e^{-}\right)$and inverse Compton scattering of produced electrons and positrons through background photons $\left(e^{ \pm} \gamma_{\mathrm{bg}} \rightarrow e^{ \pm} \gamma\right)$. These two processes produce electromagnetic showers and 
the non-thermal photon spectrum realizes a quasi-static equilibrium. The non-thermal photons experience additional processes including: Compton scattering $\left(\gamma e_{\mathrm{bg}}^{ \pm} \rightarrow \gamma e^{ \pm}\right)$; Bethe-Heitler ordinary pair creation in nuclei $\left(\gamma N_{\mathrm{bg}} \rightarrow e^{+} e^{-} N\right)$; and double photon scattering $\left(\gamma \gamma_{\mathrm{bg}} \rightarrow \gamma \gamma\right)$. These slower processes further degrade the quasi-static equilibrium photon spectrum.

This non-thermal photons might interact with background nuclei and different nuclear species are produced. The primary reactions and their cross sections we used are taken from [in. If the photo-dissociated light nucleus of a primary reaction has enough energy to induce further nuclear reactions, then secondary or tertiary processes are possible. The energy loss rates of nuclear species while propagating through the background are taken from [6.6- We also take into account the destruction of $\mathrm{D}, \mathrm{T},{ }^{3} \mathrm{He}$ and ${ }^{6} \mathrm{Li}$ after primary production by abundant background nuclides. And the relevant processes in the secondary non-thermal production of ${ }^{6} \mathrm{Li}$ involve interactions of background ${ }^{4} \mathrm{He}$ with primary tritium and ${ }^{3} \mathrm{He}$ particles. We have taken into account these two reactions with their cross sections from [편] $]$.

\section{Observations of Light Element Abundances}

\subsection{Light element abundances}

The primordial abundances of $\mathrm{D},{ }^{3} \mathrm{He},{ }^{4} \mathrm{He}$, and ${ }^{7} \mathrm{Li}$ are inferred from various observations. Here, we summarize our adopted constraints. See [9-1 for references of observational data.

$$
\begin{aligned}
1.4 \times 10^{-5}<\mathrm{D} / \mathrm{H} & <5.2 \times 10^{-5} \\
{ }^{3} \mathrm{He} / \mathrm{H} & <3.1 \times 10^{-5} \\
0.232<\quad Y \quad & <0.258 \\
1.1 \times 10^{-10}<{ }^{7} \mathrm{Li} / \mathrm{H} & <7.1 \times 10^{-10} .
\end{aligned}
$$

${ }^{6} \mathrm{Li}$ has also been measured in MPHSs by spectroscopy. In [8]1], ${ }^{6} \mathrm{Li}$ was detected at a better than two sigma significance in nine of the 24 stars observed. They suggest that a ${ }^{6} \mathrm{Li}$ plateau exists at $\log \varepsilon_{6 \mathrm{Li}} \approx 0.8$. Because the SBBN predicts much less abundance of the primordial ${ }^{6} \mathrm{Li}$ $\left({ }^{6} \mathrm{Li} /{ }^{7} \mathrm{Li} \sim 10^{-5}\right)$, some mechanism should have produced almost all ${ }^{6} \mathrm{Li}$ in MPHSs. Since multiple

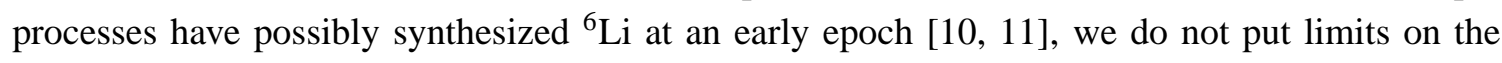
primordial abundance of ${ }^{6} \mathrm{Li}$. However, we adopt the average value of the abundance derived from the eight MPHSs with detections as a guide,

$$
{ }^{6} \mathrm{Li} / \mathrm{H} \approx 6.6 \times 10^{-12} .
$$

\subsection{Cosmic microwave background anisotropies}

Very precise data have been obtained by observations of the spectrum of temperature fluctuations in the CMB. The WMAP data have been analyzed and the energy density of baryons in the universe has been deduced, which leads to $\Omega_{b} h^{2}=0.0224 \pm 0.0009$ for the WMAP first year data [i-1] in the running scalar spectral index model. We adopt a corresponding value of $\eta=\left(6.1_{-0.2}^{+0.3}\right) \times 10^{-10}$. The SBBN with the WMAP $\Omega_{b} h^{2}$ parameter region has been calculated 
including the uncertainties of the inferred $\Omega_{b} h^{2}$ and of the reaction rates on the SBBN [1] 2i]. Their result is:

$$
\begin{aligned}
\mathrm{D} / \mathrm{H} & =\left(2.60_{-0.17}^{+0.19}\right) \times 10^{-5} \\
{ }^{3} \mathrm{He} / \mathrm{H} & =(1.04 \pm 0.04) \times 10^{-5} \\
Y & =0.2479 \pm 0.0004 \\
{ }^{7} \mathrm{Li} / \mathrm{H} & =\left(4.15_{-0.45}^{+0.49}\right) \times 10^{-10} .
\end{aligned}
$$

\section{Result}

We have calculated [99]] the cosmological nucleosynthesis including the SBBN and non-thermal nucleosynthesis induced by the radiative decay of a long-lived particle. The SBBN was computed using the Kawano code [1] $\left.{ }^{1} 3_{1}^{\prime}\right]$ with the use of the new world average of the neutron lifetime [1] checked the effect of secondary destruction of the primary non-thermal nuclides. We confirmed that the secondary destruction processes of primary nuclides were not very efficient (destruction probabilities are $\leq \mathscr{O}\left(10^{-3}\right)$ ), since the time scale of the Coulomb loss for the non-thermal nuclides is much smaller than those of the destruction reactions.

We have derived the constraints on the lifetime $\tau_{X}$ and abundance parameter $\zeta_{X}$ from the adopted limits for the cosmological light element abundances. Our result is very similar to that of [A] timated cross sections. A detailed explanation has been given in [i] radiative decay. Fig. ${ }_{-1}^{1} i_{1}^{\prime}$ shows the derived constraint on $\tau_{X}$ and $\zeta_{X}$ for an unstable particle from the above consideration of the light element abundances in a model with $\eta=6.1 \times 10^{-10}$. The ${ }^{3} \mathrm{He}$ overabundant region is shaded by the dark gray, and the rest of the excluded region the light gray. The light colored region is fixed largely by the deuterium underproduction. For $\tau_{X} \gtrsim 10^{6} \mathrm{~s},{ }^{3} \mathrm{He}$ provides the strongest limit on the abundance parameter, while for shorter lifetimes $\left(\tau_{X} \sim 10^{4}-10^{6} \mathrm{~s}\right)$ the limits are from $\mathrm{D}$, implying $\zeta_{X} \underset{\sim}{\sim} 10^{-9} \mathrm{GeV}$.

\section{Discussion}

\subsection{Distortion of the CMB spectrum}

Since non-thermal photons produced by the radiative decay deform the blackbody spectrum of the CMB, this is limited by the consistency of the observed CMB data with a blackbody spec-

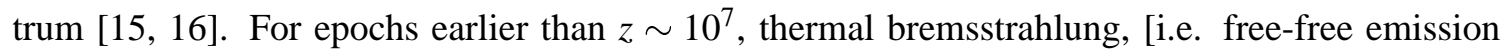
$(e N \rightarrow e N \gamma)$, where $N$ is an ion] and radiative-Compton scattering $\left(e^{-} \gamma \rightarrow e^{-} \gamma \gamma\right)$ act effectively to erase any distortion of the CBR spectrum from a blackbody. For the decay in epochs $10^{5}<z<10^{7}$, processes changing the photon number become ineffective, and Compton scattering $\left(\gamma e^{-} \rightarrow \gamma e^{-}\right)$ causes the photons and electrons to achieve statistical equilibrium. Then, the photon spectrum should have a Bose-Einstein distribution

$$
f_{\gamma}\left(\vec{p}_{\gamma}\right)=\frac{1}{e^{\varepsilon_{\gamma} / T+\mu}-1},
$$



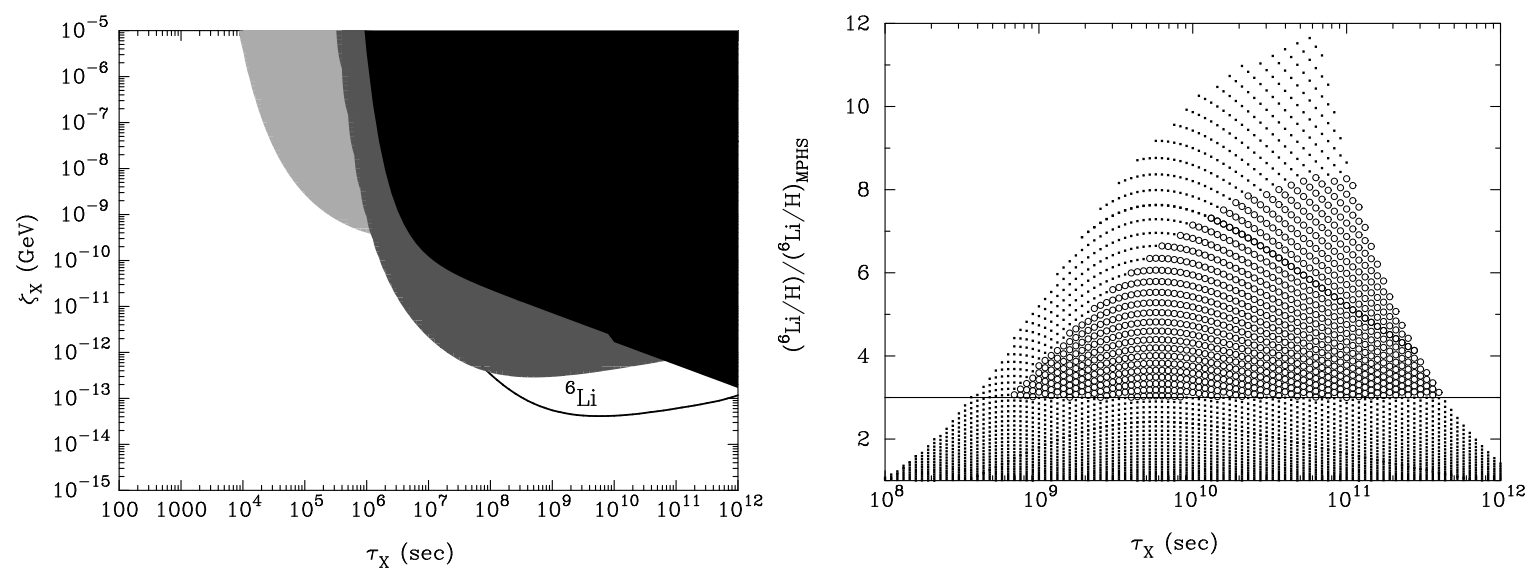

Figure 1: Gray regions identify the excluded area in Figure 2: Ratio of calculated ${ }^{6} \mathrm{Li} / \mathrm{H}$ abundances (afthe parameter space $\left(\tau_{X}, \zeta_{X}\right)$ for models with a fixed ter the non-thermal nucleosynthesis) to the observed value of $\eta=6.1 \times 10^{-10}$. The dark gray region is ex- abundance in MPHSs as a function of $\tau_{X}$. Results cluded by an overabundance of ${ }^{3} \mathrm{He}$, whereas the light in the allowed parameter region of $\left(\tau_{X}, \zeta_{X}\right)$ producshaded region is mostly excluded by an underabun- ing ${ }^{6} \mathrm{Li} / \mathrm{H}$ larger than the value found in MPHSs, or dance of deuterium. The black shaded region super- the marked region "6 Li" in Fig. '11', are plotted. The imposed shows the region excluded by the consistency horizontal line indicates a factor of three overproducrequirement of the $\mathrm{CMB}$ with a blackbody. The curved tion of ${ }^{6} \mathrm{Li}$ relative to the observed MPHS value of line identifies the contour of ${ }^{6} \mathrm{Li} / \mathrm{H}=6.6 \times 10^{-12},{ }^{6} \mathrm{Li} / \mathrm{H}=3 \times 6.6 \times 10^{-12}$. The large circles denote valcorresponding to the abundance of ${ }^{6} \mathrm{Li}$ observed in ues in the allowed region with abundances of ${ }^{3} \mathrm{He} / \mathrm{H}=$ MPHSs. The region above the contour and below the $1.3-2.5 \times 10^{-5}$ and ${ }^{6} \mathrm{Li} / \mathrm{H} \geq 3 \times 6.6 \times 10^{-12}$. The nucleosynthesis and CMB constraints is allowed and other parameters sets are indicated by small squares. abundant in ${ }^{6} \mathrm{Li}$. This figure is taken from [ị̂n].

This figure is taken from [9,9].

where $\mu$ is the dimensionless chemical potential derived from the conservation of photon number. Analyses of the CMB data suggest a relatively low baryon density so that radiative-Compton scattering dominates the thermalization process. For small energy injection from the radiative decay, the chemical potential can be approximated analytically [1] $\left.\overline{1}_{1}^{1}\right]$.

For a late energy injection at $z<10^{5}$, Compton scattering produces little effect and cannot establish a Bose-Einstein spectrum. The distorted spectrum is then described by the Compton $y$ parameter. There is a relation between $y$ and the amount of the injected energy, $\Delta E / E_{\mathrm{CBR}}=4 y$, where $\Delta E$ and $E_{\mathrm{CBR}}$ are the total energy injected and the CBR energy, respectively.

The CMB spectrum has been well measured and the deduced limits are $|\mu|<9 \times 10^{-5},|y|<$ $1.2 \times 10^{-5}$ [1] region of $\zeta_{X}$ is excluded by the $\mu$ and $y$ limits. In Fig. '1' the black shading indicates the parameter region excluded by the CBR distortion limit. For a lifetime shorter than $\tau_{X}=4 \times 10^{11} \mathrm{~s} \Omega_{b} h^{2} \sim$ $8.8 \times 10^{9} \mathrm{~s}$, the decay is constrained by the chemical potential $\mu$. On the other hand, when an unstable particle decays later, the CBR spectrum is limited by the Compton $y$ parameter. The parameter region of relatively long lifetime $\left(10^{10} \mathrm{~s}<\tau_{X}\right)$ is constrained by the CMB spectrum more strongly than the light element abundances.

\subsection{Parameter region consistent with ${ }^{6} \mathrm{Li}$ in MPHSs}

We analyze the possibility that the radiative decay of long-lived particles produces ${ }^{6} \mathrm{Li}$ by 
non-thermal process while having almost no effect on ${ }^{7} \mathrm{Li}$ or other nuclides produced in the SBBN. Ellis, Olive \& Vangioni studied the possibility that the radiative decay of unstable particles explains the discrepancy of the BBN calculated ${ }^{7} \mathrm{Li}$ abundance and low ${ }^{7} \mathrm{Li}$ plateau derived from observations [1] $1 \overline{1}]$. They found that in the parameter region where ${ }^{7} \mathrm{Li}$ is photo-dissociated down to the level of the ${ }^{7} \mathrm{Li}$ plateau, either the $\mathrm{D}$ abundance was too low or the ratio ${ }^{3} \mathrm{He} / \mathrm{D}$ was too large in the context of standard stellar evolution and chemical evolution. They concluded that radiative particle decays cannot be a cause for the ${ }^{7} \mathrm{Li}$ abundance difference. They also mentioned the possibility of ${ }^{6} \mathrm{Li}$ production in their paper.

Uncertainties remain in estimations of the $\mathrm{Li}$ abundance in stellar atmospheres, and the probability of depletion in stars has not been excluded. Therefore, we suppose that the discrepancy of the ${ }^{7} \mathrm{Li}$ abundance is caused by stellar depletion or some other systematic effect. Then the ${ }^{6} \mathrm{Li}$ abundance in the early universe should have been larger when first engulfed in a star than the value presently deduced from observations of MPHSs. Assuming that is the case, we impose the following constraint on the ${ }^{6} \mathrm{Li}$ abundance after the radiative decay process,

$$
{ }^{6} \mathrm{Li} / \mathrm{H}>6.6 \times 10^{-12} .
$$

In Fig. $1_{1}^{1}$ the contour of the lower limit $(\overline{5} .2)$ is shown by a solid line below the CMB constraint. Hence, a ${ }^{6} \mathrm{Li}$-producing allowed parameter region certainly exists for $\tau_{X}=10^{8}-10^{12} \mathrm{~s}$ and $\zeta_{X} \sim$ $10^{-13}-10^{-12} \mathrm{GeV}$. The parameter region allowed by the above constraints which also produces abundant ${ }^{6} \mathrm{Li}$ is marked as "6 $\mathrm{Li}$ ".

We have analyzed this parameter region to see the possibility of realization. We pick up a model calculation with input parameters of $\tau_{X}=1 \times 10^{10} \mathrm{~s}, \zeta_{X}=3 \times 10^{-13} \mathrm{GeV}$ and $\eta=6.1 \times$ $10^{-10}$. The final abundances obtained in this model are

$$
\begin{aligned}
\mathrm{D} / \mathrm{H} & =2.63 \times 10^{-5} \\
{ }^{3} \mathrm{He} / \mathrm{H} & =2.48 \times 10^{-5} \\
Y & =0.247 \\
{ }^{6} \mathrm{Li} / \mathrm{H} & =4.69 \times 10^{-11} \\
{ }^{7} \mathrm{Li} / \mathrm{H} & =4.36 \times 10^{-10} .
\end{aligned}
$$

These are certainly consistent with the constraints we adopted in Sec. 1 . The abundances of ${ }^{3} \mathrm{He}$ and ${ }^{6} \mathrm{Li}$ with respect to the SBBN abundances increase. The non-thermal ${ }^{6} \mathrm{Li}$ production inevitably brings about the production of ${ }^{3} \mathrm{He}$, and this gives a strong constraint on the possible

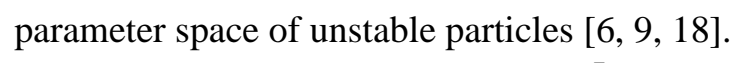

If the inconsistency between the ${ }^{7} \mathrm{Li}$ abundance predicted by SBBN and that measured from MPHSs is caused by stellar depletion, ${ }^{6} \mathrm{Li}$ would have existed in the primordial gas at a level larger than the abundance observed in MPHSs by at least the ratio of the $\mathrm{SBBN}{ }^{7} \mathrm{Li} / \mathrm{H}$ prediction to the mean value observed in MPHSs. The observed ${ }^{7} \mathrm{Li} / \mathrm{H}$ abundance [i, is is ${ }^{7} \mathrm{Li} / \mathrm{H} \sim 1.62 \times 10^{-10}$. Hence, this factor is $\sim 3$. So ${ }^{6} \mathrm{Li}$ should have been originally produced at an abundance more than about 3 times the presently observed value.

We have analyzed the upper limit to the ${ }^{6} \mathrm{Li}$ abundance resulting from the radiative decay process under the requirement of consistency with the other light-element abundances. In Fig. 2 is the 
${ }^{6} \mathrm{Li}$ abundances are plotted as a function of $\tau_{X}$. Points on this figure are allowed by the constraints imposed above and lead to ${ }^{6} \mathrm{Li}$ abundances above the level observed in MPHSs. The vertical scale is ${ }^{6} \mathrm{Li} / \mathrm{H}$ normalized to the mean ${ }^{6} \mathrm{Li} / \mathrm{H}$ abundance in MPHSs $\left({ }^{6} \mathrm{Li} / \mathrm{H}\right)_{\mathrm{MPHS}}$. The horizontal line indicates a factor of three enhancement in ${ }^{6} \mathrm{Li}$. The large circles are for cases with more than three times as abundant ${ }^{6} \mathrm{Li}$ as the level found in MPHSs. Here, we adopt the one sigma ${ }^{3} \mathrm{He} / \mathrm{H}=(1.9 \pm 0.6) \times 10^{-5}\left[{ }_{1}^{1} \overline{1}_{9-1}^{\top}\right]$ as an extra constraint. We note that, in a case adopting a tighter constraint ${ }^{3} \mathrm{He} / \mathrm{H}<(1.6 \pm 0.3) \times 10^{-5}\left[{ }_{2}^{2} \overline{2}_{-1}^{\prime}\right]$, one can still find an allowed region of $\tau_{X}=3 \times 10^{10}$ $3 \times 10^{11} \mathrm{~s}$ which satisfies the same constraint imposed on the ${ }^{6} \mathrm{Li}$ abundance. The small squares are for the other case of Eq. (3.3).

This figure confirms that ${ }^{6} \mathrm{Li} / \mathrm{H}$ abundances as large as those in MPHSs multiplied by the ratio $\left({ }^{7} \mathrm{Li} / \mathrm{H}\right) /\left({ }^{7} \mathrm{Li} / \mathrm{H}\right)_{\mathrm{MPHS}}$ can be produced by non-thermal nucleosynthesis without significantly impacting the other nuclide abundances. Although this explanation could resolve the discrepancy between the SBBN predicted ${ }^{6} \mathrm{Li}$ abundances and those derived from observations, it cannot resolve the so-called lithium problem. This scenario necessarily requires some model for the stellar depletion of ${ }^{6} \mathrm{Li}$ and ${ }^{7} \mathrm{Li}$. Indeed, as discussed in [8.] and references therein, models exist which suggest a very large depletion factor of ${ }^{6} \mathrm{Li}$ along with some ${ }^{7} \mathrm{Li}$ depletion. The production of ${ }^{6} \mathrm{Li}$ by radiative decay cannot explain the observed abundances of both ${ }^{6} \mathrm{Li}$ and ${ }^{7} \mathrm{Li}$, if the stellar depletion proceeds as described by that model. However, approximately equal amounts of depletion for both lithium isotopes can explain the measured abundances when combined with the non-thermal production of ${ }^{6} \mathrm{Li}[\overline{9}]$ ] that such particle decay could simultaneously solve both the ${ }^{6} \mathrm{Li}$ and ${ }^{7} \mathrm{Li}$ problem, even if a possible degree of depletion is included.

\section{References}

[1] D. N. Spergel et al. [WMAP Collaboration], First Year Wilkinson Microwave Anisotropy Probe (WMAP) Observations: Determination of Cosmological Parameters, ApJS 148, 175 (2003), [astro-ph/0302209]

[2] D. N. Spergel et al., Wilkinson Microwave Anisotropy Probe (WMAP) three year results: Implications for cosmology, astro-ph/0603449.

[3] K. Jedamzik, Lithium-6: A Probe of the Early Universe, Phys. Rev. Lett. 84, 3248 (2000), [astro-ph/9909445].

[4] R. H. Cyburt, J. Ellis, B. D. Fields and K. A. Olive, Updated nucleosynthesis constraints on unstable relic particles, Phys. Rev. D 67, 103521 (2003), [astro-ph/0211258].

[5] K. Jedamzik, Did something decay, evaporate, or annihilate during big bang nucleosynthesis?, Phys. Rev. D 70, 063524 (2004), [astro-ph/ 0402344 ].

[6] M. Kawasaki, K. Kohri and T. Moroi, Big-bang nucleosynthesis and hadronic decay of long-lived massive particles, Phys. Rev. D 71, 083502 (2005), [ast ro-ph/ 0408426 ].

[7] S. G. Ryan, T. C. Beers, K. A. Olive, B. D. Fields and J. E. Norris, Primordial Lithium and Big Bang Nucleosynthesis, ApJ 530, L57 (2000), [astro-ph/9905211].

[8] M. Asplund, D. L. Lambert, P. E. Nissen, F. Primas and V. V. Smith, Lithium isotopic abundances in metal-poor halo stars, ApJ 644, 229 (2006), [astro-ph/ 0510636]. 
[9] M. Kusakabe, T. Kajino and G. J. Mathews, ${ }^{6}$ Li Production by the Radiative Decay of Long-Lived Particles, Phys. Rev. D 74, 023526 (2006), [astro-ph/ 0605255$].$

[10] T. K. Suzuki and S. Inoue, Cosmic-Ray Production of ${ }^{6}$ Li by Structure Formation Shocks in the Early Milky Way: A Fossil Record of Dissipative Processes during Galaxy Formation, ApJ 573, 168 (2002), [astro-ph/0201190].

[11] E. Rollinde, E. Vangioni and K. A. Olive, Population III Generated Cosmic Rays and the Production of Li6, astro-ph/0605633.

[12] A. Coc, E. Vangioni-Flam, P. Descouvemont, A. Adahchour and C. Angulo, Updated Big Bang Nucleosynthesis confronted to WMAP observations and to the Abundance of Light Elements, ApJ 600, 544 (2004), [astro-ph/0309480]

[13] L. H. Kawano, preprint FERMILAB-Pub-92/04-A (1992)

[14] G. J. Mathews, T. Kajino and T. Shima, Big Bang Nucleosynthesis with a New Neutron Lifetime, Phys. Rev. D 71, 021302(R) (2005), [astro-ph/ 0408523 ].

[15] W. Hu and J. Silk, Thermalization constraints and spectral distortions for massive unstable relic particles, Phys. Rev. Lett. 70, 2661 (1993)

[16] J. L. Feng, A. Rajaraman and F. Takayama, Superweakly-interacting massive particles, Phys. Rev. Lett. 91, 011302 (2003), [hep-ph/0302215].

[17] K. Hagiwara et al. [Particle Data Group], Review of particle physics, Phys. Rev. D 66, 010001 (2002)

[18] J. R. Ellis, K. A. Olive and E. Vangioni, Effects of unstable particles on light-element abundances: Lithium versus deuterium and He-3, Phys. Lett. B 619, 30 (2005), [astro-ph/ 0503023 ].

[19] T. M. Bania, R. T. Rood and D. S. Balser, The cosmological density of baryons from observations of ${ }^{3} \mathrm{He}^{+}$in the Milky Way, Nature 415, 54 (2002)

[20] H. Busemann et al., Interstellar Helium Trapped with the COLLISA Experiment on the MiR Space Station-Improved Isotope Analysis by In Vacuo Etching, ApJ 639, 246 (2006)

[21] K. Jedamzik, K. Y. Choi, L. Roszkowski and R. Ruiz de Austri, Solving the cosmic lithium problems with gravitino dark matter in the CMSSM, hep-ph/0512044. 